\title{
Indirect Search for Dark Matter from the Galactic Center with the Fermi Gamma Ray Satellite
}

\author{
Vincenzo Vitale ${ }^{* 1,2}$, Aldo Morselli ${ }^{2}$, Beatriz Canadas ${ }^{1,3}$ for the Fermi-LAT \\ Collaboration \\ (1) Dipartimento di Fisica, Univ. Roma Tor Vergata \\ (2) Sezione INFN Roma Tor Vergata \\ (3) Univ. Autonoma Madrid \\ E-mail: vincenzo.vitaleeroma2.infn.it
}

High energy gamma ray observations can be used for the indirect search for annihilating Dark Matter. One of the possible observation strategy is the targeting of regions where high Dark Matter density is foreseen, such as the Galactic Center. This observation poses a challenge: to disentangle the possible Dark Matter signal from the bright gamma radiation of astrophysical sources. The Large Area Telescope on board of the GLAST satellite provides the best opportunity to date for such a study, because of its excellent sensitivity and angular resolution, as compared to the past satellite-borne detectors. A report of the GLAST LAT Dark Matter Group activities on this topic will be given.

Identification of dark matter 2008

August 18-22, 2008

Stockholm, Sweden

${ }^{*}$ Speaker. 


\section{The Indirect Search for Dark Matter from the Galactic Center}

The estimated Universe energy content [1] is : 4\% of baryonic ordinary matter, 23\% of Dark Matter (DM) and 73\% of Dark Energy. DM is gravitationally coupled with ordinary matter, other details of DM nature are unknown. There are several evidences of the DM existence: galaxies rotation velocities [2]; galaxies orbital velocities within clusters[3]; gravitational lensing [4]; the cosmic microwave background [5]; light elements abundances [6]; and large scale structures [7]. Non-gravitational DM couplings are studied with: (1) the search for DM scattering on ordinary matter; (2) the indirect study of DM annihilation via the secondary products, both charged and neutral $\left(\mathrm{e}^{+}, \bar{p}, \bar{d}, v, \gamma\right.$ rays and lower frequency electro-magnetic radiation), if DM particles selfannihilate and produce quarks, leptons and gauge bosons. The annihilating DM $\gamma$-ray flux, from the Galactic Center (GC) can be expressed as: $\Phi_{D M}=\sum b_{i} \frac{d N_{\gamma, i}}{d E_{\gamma}} \frac{\sigma v}{8 \pi m_{X}^{2}} \int_{l o s} \rho^{2}(l) d l$ where $\sigma \mathrm{v}$ is the DM annihilation cross section times the relative particles velocities, $\mathrm{m}_{X}=\mathrm{DM}$ particle mass, $\rho(\mathrm{r})=\mathrm{DM}$ density as a function GC distance, the integral is performed along the line-of-sight, $\frac{d N_{\gamma}}{d E_{\gamma}}=$ annihilation $\gamma$-ray yield and $\mathrm{b}_{i}$ the braching ratio. DM forms halos with central density enhancements and the Milky Way is embedded in one of such structures. The galactic density profile is commonly parametrized as $\rho(r)=\frac{\rho_{0}}{(r / R)^{\gamma}\left(1+(r / R)^{\alpha}\right)^{(\beta-\gamma) / \alpha}}$ with $\mathrm{R} \approx 20 \mathrm{kpc}$ as scale radius, $\rho_{0}$ fixed such as the DM density $=0.3 \mathrm{GeV} / \mathrm{cm}^{3}$ in the Sun region. The other three parameters are defining the profile type: Navarro-Frenk-White (NFW, [8]) has $\alpha=1, \beta=3, \gamma=1$; Moore profile [9] has $\alpha=1.5 \beta=3, \gamma=1.5$. The density profile is essential for the DM indirect study in the $\gamma$ ray channel as:(1) the $\gamma$-ray flux goes as $\rho^{2}$ and can be above or below the detection threshold as function of the profile type; (2) some density profiles might be detected as extended sources. The spatial information and the peculiar energy spectrum [20], expected by a DM source, will be fundamental in the GC region, where bright $\gamma$-ray sources are located. The Fermi Gamma Ray satellite, reported in [10], the most sensitive instrument in the $20 \mathrm{MeV}-300 \mathrm{GeV}$ band, is well suited for the indirect DM investigations (see also [11], [12], [13]).

\section{Simulation of the DM Signal in the Galactic Center and the Background}

The Fermi Gamma Ray Satellite sensitivity for DM indirect searches was investigated in [14]. For the GC the DM mass was considered between 10 and $1000 \mathrm{GeV}$, while the $\sigma \mathrm{v}$ parameter between 0.5 and $100 \times 10^{-26} \mathrm{~cm}^{-3} \mathrm{~s}^{-1}$. The Galactic diffuse emission (both conventional and optimized GALPROP models, see ref. in [14]) was assumed as background and a $\chi^{2}$ analysis was applied. In the present study we used: the Galactic Diffuse emission and the main known astrophysical $\gamma$-ray sources as background. Furthermore the Maximum Likelyhood Analysis was applied, since this analysis type is sensitive to the source spatial distribution. The main $\gamma$-ray sources detected in the GC region so far, with EGRET and IACTs are: (A) 3EG J1746-2851, an unidentified EGRET source, close to $\operatorname{SgrA}^{*}$ [15], with a flux $\mathrm{F}(\mathrm{E}>100 \mathrm{MeV})=(49 \pm 3) \times 10^{-8} \mathrm{~cm}^{-2} \mathrm{~s}^{-1}$. The source was also interpreted as a possible DM $\gamma$-ray emission in [16]; (B) the TeV source in the Galactic Center (HESS J1745-290), a bright source above $100 \mathrm{GeV}$, detected by many IACTs, most recently by HESS [17] and MAGIC [18]. The source has a steady flux with a power-law 

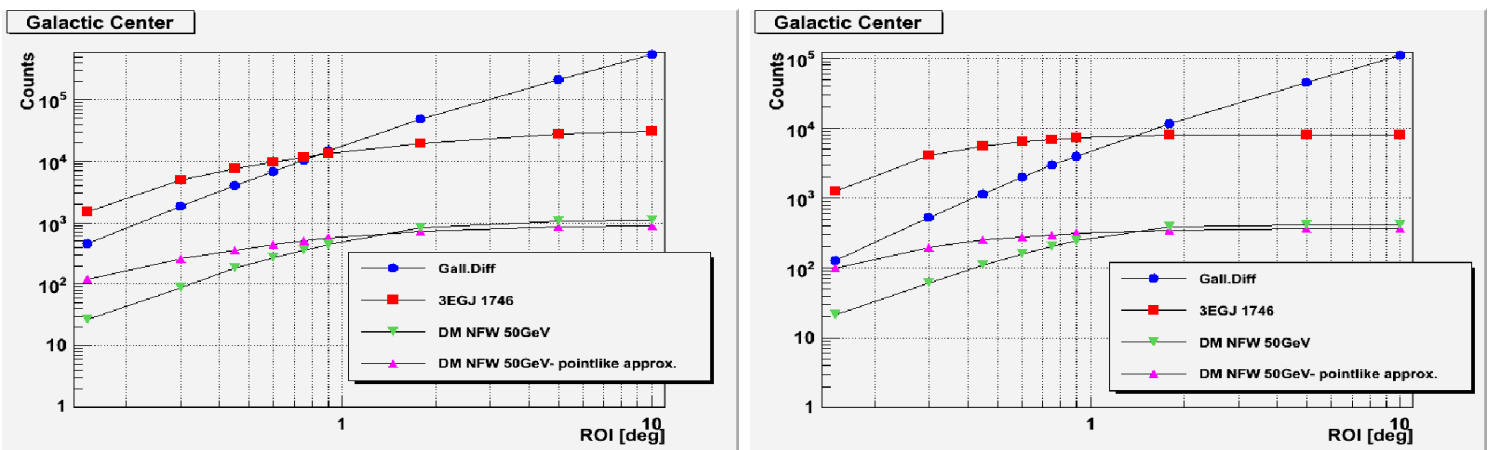

Figure 1: Counts versus ROI radius, for various simulations components. Events above $100 \mathrm{MeV}$ on the left, above $1 \mathrm{GeV}$ on the right. Major differences below 1.8 degrees are shown between a DM source with a NFW spatial distribution and one with point-like approximation.
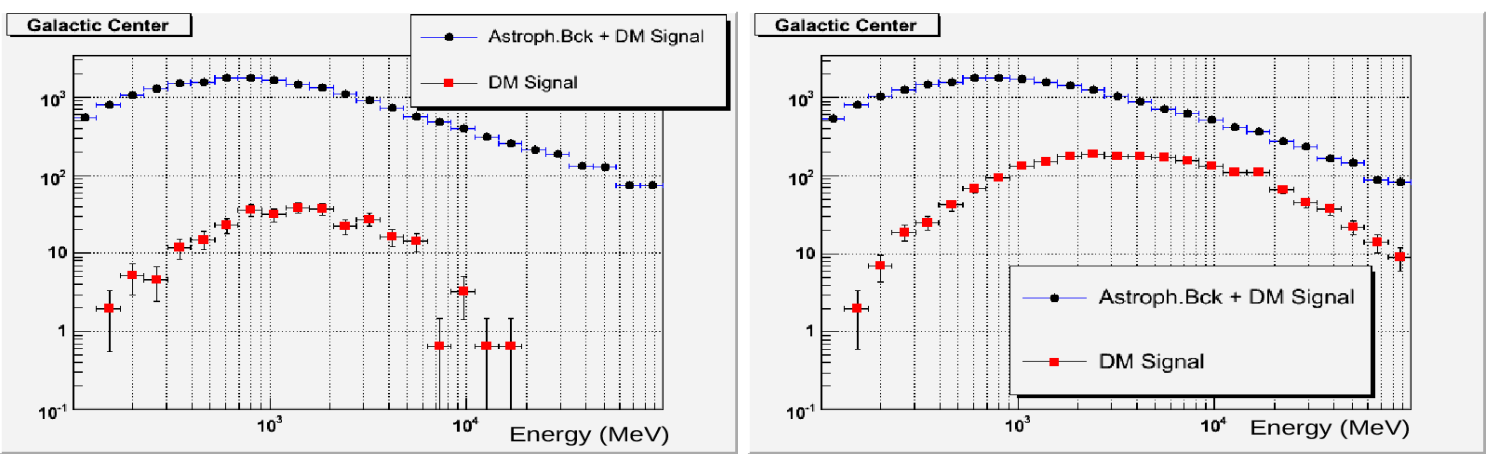

Figure 2: Counts versus Energy, for two realizations of a DM source. DM particles with mass of $50 \mathrm{GeV}$ and a NFW spatial distribution on the left, mass of $500 \mathrm{GeV}$ and an adiabatically contracted NFW distribution on the right.

spectrum ( $\Gamma=2.25 \pm 0.04)$. HESS data disfavour an interpretation of DM $\gamma$-ray emission, if DM with mass above $10 \mathrm{TeV}$ is not considered. Furthermore many possible astrophysical counterparts of the source are considered; (C) the TeV Diffuse Emission discovered with HESS [19], possibly associated with dense molecular clouds in the GC region. (D) other $\gamma$ sources such as 3EG J17362908, 3EG J 1744-3011 (HESS J1745-303 ?), HESS J1747-281(G0.9-0.1), 3EG J1746-2851 (see also [20]). The sources A,B and $\mathrm{C}$ were modelled and included in Fermi observation simulations together with the GALPROP Diffuse Emission models. The DM source was modelled with: the DarkSusy code for the spectrum (DM mass $=50,241$ and $500 \mathrm{GeV}, \sigma \mathrm{v}=3 \times 10^{-26} \mathrm{~cm}^{-3} \mathrm{~s}^{-1}, b \bar{b}$ channel); the NFW and the Adiabatic NFW [21] for the source spatial distribution.

\section{Likelihood Analysis and Results}

A signal enhancement can be obtained with the Region-of-Interest (likelihood analysis region) optimization. In Fig 1 the counts versus the ROI radius are shown for various simulation components. An optimal ROI of $1.8^{\circ}$ for the DM source was found when the optimization function $f=$ $\frac{D M}{\sqrt{D M+\text { background }}}$ was used. A more realistic result can be given with the likelihood TS parameter as 
optimization function. For each DM source realization the absolute number of detected $\gamma$ rays was obtaind (Fig 2). For example in 30Ms, a DM source with DM mass $=50 \mathrm{GeV}$ and NFW profile (DM50NFW) yielded 280 counts, a DM source with DM mass $=500 \mathrm{GeV}$ and Adiabatic NFW profile (DM500ADB) yielded 2136. In the same time 19710 background counts were detected. The likelihood analysis was applied by means of the Fermi analysis tools. First it was applied to single components, then to the GC region with all the simulated emissions. The fit parameters of the main sources were fixed. With real data, the DM search requires thorough understanding of the involved astrophysical backgrounds. Some of the benchmark DM sources were then detected, for example DM50NFW was detected above $5 \sigma$ in $30 \mathrm{Ms}$, while DM500ADB at $9 \sigma$ in $6 \mathrm{Ms}$. These relatively realistic simulations demonstrate: (1) the likelihood analysis is well suited for the GC $\gamma$-ray study; (2) for a couple of DM benchmarks the detection is feasable within one year.

\section{References}

[1] E. Komatsu et. al. arXiv 0803.0547

[2] A.Borriello et al., MNRAS 323, 285 (2001)

[3] F.Zwicky, Helv.Phys. Acta 6, 110 (1933)

[4] J.A. Tyson et al., Astroph.J. 498, L107 (1998)

[5] D.N. Spergel et al., Astroph.J.Suppl. 170, 377 (2007)

[6] K.A. Olive et al., Phys.Rept. 333, 389 (2000)

[7] M. Tegmark et al., Astroph. J. 606, 702 (2004)

[8] J.F.Navarro et al., Astroph.J. 462, 563 (1996)

[9] B. Moore et al., Astroph. J. 524, L19 (1999)

[10] J. Cohen-Tanugi et al, these proceedings

[11] E.Nuss et al., these proceedings

[12] T. Ylinen et al., these proceedings

[13] C. Meurer, to be published in AIP Conference Proceedings (2008)

[14] E.A. Baltz et al., JCAP 7, 13 (2008)

[15] Mayer-Hasselwander et al., A\&A 335, 161 (1998)

[16] A. Morselli et al., AstroPart. Phys. 21, 267 (2004)

[17] F. Aharonian et al., A\&A 425, L13 (2005)

[18] J. Albert et al., Astroph.J. 639, 761 (2006)

[19] F. Aharonian et al., NATURE 439, 695 (2006)

[20] S. Profumo et al., arXiv 0808.2641 (2008)

[21] J.F.Navarro et al.,MNRAS 349, 1039 